TITLE:

\title{
Conflict, growth, distribution, and employment: a long-run Kaleckian model
}

\author{
AUTHOR(S):
}

Sasaki, Hiroaki

\section{CITATION:}

Sasaki, Hiroaki. Conflict, growth, distribution, and employment: a long-run Kaleckian model. International Review of Applied Economics 2011, 25(5): 539-557

\section{ISSUE DATE:}

2011-09

URL:

http://hdl.handle.net/2433/197369

\section{RIGHT:}

The Version of Record of this manuscript has been published and is available in International Review of Applied Economics (2011)

http://www.tandfonline.com/10.1080/02692171.2011.557057; この論文は出版社版であり ません。引用の際には出版社版をご確認ご利用ください。; This is not the published version. Please cite only the published version. 


\title{
Conflict, Growth, Distribution, and Employment: A Long-Run Kaleckian Model
}

\author{
Hiroaki SASAKI \\ Graduate School of Economics and Management, Tohoku University
}

\begin{abstract}
This paper presents a Kaleckian growth model in which (i) the rate of capacity utilization is adjusted in the short run, (ii) the profit share and the rate of employment are adjusted in the medium run, and (iii) the normal rate of capacity utilization and the expected rate of capital accumulation are adjusted in the long run. The long-run equilibrium is a continuum of equilibria and is characterized by hysteresis in that the long-run position of the economy depends on where it starts. An increase in the bargaining power of workers lowers the rate of unemployment in both the medium-run and the long-run equilibrium.
\end{abstract}

Keywords: Kaleckian model; long-run equilibrium; unemployment; path-dependency JEL Classification: E12; E22; E24; E25; O41

\section{Introduction}

This paper develops a Kaleckian growth model in which the rate of employment is endogenously determined. Using the model, we investigate the short-run, medium-run, and long-run equilibria.

Thus far, a number of Kaleckian models have been developed and improved. ${ }^{1}$ Kaleckian models have the following four characteristics (Lavoie, 1992, 1995): (1) the investment function; (2) prices relative to direct costs are influenced by a broad range of factors, often summarized under the phrase "degree of monopoly"; (3) marginal costs are assumed to be constant up to full capacity; and (4) the rate of capacity utilization is assumed to be generally below unity. In early Kaleckian models, the rate of capacity utilization and the rate of capital accumulation are determined with income distribution given exogenously. New Kaleckian models were then proposed in which income distribution is endogenously determined with the theory of conflicting-claims inflation. ${ }^{2}$

However, Marxists and Sraffians criticize the Kaleckian model, claiming that the Kaleckian 
model is a short-run, medium-run model and not a long-run model (Auerbach and Skott, 1988; Duménil and Lévy, 1999; Park, 1997). In the Kaleckian model, the equilibrium rate of capacity utilization diverges from the normal rate of capacity utilization. Critics assert that this divergence does not last and should vanish in the long run; accordingly, the Kaleckian model lacks logical consistency. For Marxists and Sraffians, the long-run equilibrium is a state where all variables are fully adjusted.

In contrast to this criticism, Lavoie $(1995,2002,2003)$ and Cassetti (2006) introduce the adjustment process of the normal rate of capacity utilization in the long run. ${ }^{3}$ They show that even in the long-run equilibrium where the actual rate of capacity utilization and the normal rate of capacity utilization are equalized, "the paradox thrift" and "the paradox of cost" hold, which are important characteristics of the Kaleckian model. Here, the paradox of thrift means that an increase in capitalists' propensity to save lowers the rate of capital accumulation. The paradox of cost means that an increase in the real wage leads to an increase in the realized profit rate.

However, even such extended models miss an important point, that is, the determination of the rate of employment, which we focus on in this paper. Conventional Kaleckian growth models assume that labor supply is unlimited and that firms employ as many workers as they desire at given wages. If, however, the labor supply grows at an exogenously given rate, there is no guarantee that the endogenously determined growth rate of employment would be equal to the growth rate of labor supply. Thus, if the growth of labor supply steadily exceeds that of the labor demand, then the rate of unemployment will keep on increasing; however, this is unrealistic. ${ }^{4}$

Therefore, we extend the Kaleckian model to determine the rate of employment, and to investigate how changes in parameters affect the rate of employment.

Our paper is not an initial attempt to explicitly consider the determination of the rate of employment in the Kaleckian model. Stockhammer (2004) presents an augmented Kaleckian model that incorporates equations that determine employment and income distribution, and investigates the rate of employment in the long-run equilibrium. However, the notion of the long run in Stockhammer (2004) is different from that in the present paper. The long run in Stockhammer's model corresponds to the medium run in our model. Moreover, we determine employment and income distribution differently, and thus, we obtain different results from 
those obtained by Stockhammer.

To determine the rate of employment, we endogenize the growth rate of labor productivity, which is given exogenously in conventional Kaleckian models. ${ }^{5}$ We assume that the growth rate of labor productivity depends positively on the level of employment rate. Such a formulation is also proposed by Bhaduri (2006) and Dutt (2006). Based on the idea of Marx, Bhaduri states that this captures a view that technological change is driven by inter-class conflict over income distribution between workers and capitalists. Dutt says that as the labor market tightens and a labor shortage becomes clear, the bargaining power of workers increases, which exerts an upward pressure on wages, leading capitalists to adopt labor-saving technical changes. ${ }^{6}$ Bhaduri's (2006) model is not a Kaleckian one because income distribution is not determined by mark-up pricing. However, it bears similarity to the Kaleckian model in that effective demand plays a crucial role in determining output. In contrast, Dutt's (2006) model can be said to be Kaleckian, but it does not deal with issues such as income distribution or inflation because its purpose is to present a simple growth model that integrates the roles of aggregate demand and supply.

In determining technological change, mainstream growth theory emphasizes supply side factors such as R\&D investment and human capital accumulation. In contrast, we emphasize on the demand side factors contributing to technological change: changes in aggregate demand cause changes in employment, which lead to technological change. Under our formulation, "the natural rate of growth" becomes an endogenous variable. Here, we define the natural rate of growth as a sum of the growth rate of labor productivity and that of labor supply. Although the growth rate of labor supply in our model is exogenously given, the growth rate of labor productivity is endogenously determined, and consequently, the natural rate of growth is an endogenous variable. Our formulation suggests that the natural rate of growth increases when business is good (i.e., when the rate of employment is high), while it decreases when business is bad (i.e., when the rate of employment is low). This property is consistent with the empirical studies of León-Ledesma and Thirlwall (2002), Libânio (2009), and Vogel (2009). Therefore, our formulation of technological change is reasonable in that it describes an important feature of the real world.

The framework of our model is based on Cassetti (2006). He introduces the theory of conflicting-claims inflation into the Kaleckian model and analyzes the medium-run and 
long-run equilibria. We introduce the abovementioned endogenous technical change into Cassetti's (2006) model and analyze the short-run, medium-run, and long-run equilibria. In the short run, the rate of capacity utilization is adjusted. In the medium run, the profit share and the rate of employment are adjusted. ${ }^{7}$ In the long run, the normal rate of capacity utilization and the expected rate of capital accumulation are adjusted.

The remainder of the paper is organized as follows. Section 2 presents the basic framework of our model and analyzes the short-run equilibrium. Section 3 conducts a medium-run analysis. Section 4 conducts a long-run analysis and also presents numerical examples. Section 5 concludes the paper.

\section{Short-run analysis}

\subsection{Basic framework of the model}

Consider an economy with workers and capitalists. Suppose that workers consume all their wages and capitalists save a fraction $s$ of their profits. Then, the ratio of the real saving $S$ to the capital stock $K$, that is, $g_{s}=S / K$ leads to

$$
g_{s}=s r
$$

where $r$ denotes the rate of profit.

Suppose that firms operate with the following fixed coefficients production function:

$$
Y=\min \{a E,(u / k) K\},
$$

where $Y$ denotes real output; $E$, employment; and $a=Y / E$, the level of labor productivity. ${ }^{8}$ The rate of capacity utilization is defined as $u=Y / Y^{*}$, where $Y^{*}$ denotes the potential output. The coefficient $k=K / Y^{*}$ denotes the ratio of the capital stock to the potential output, which is assumed to be constant. This assumption means that both $K$ and $Y^{*}$ grow at the same rate. Moreover, when the rate of capacity utilization is constant, the growth rate of capital stock and that of the actual output will be the same. Accordingly, the actual output and the potential output grow at the same rate in the equilibrium where the rate of capacity utilization is constant. To simplify the analysis, we assume $k=1$ in what follows. From this, we have $r=m u$, where $m$ denotes the profit share.

Let us introduce the investment function. Following Amadeo (1986) and Lavoie (2006), we specify the ratio of the real investment $I$ to the capital stock, $g_{d}=I / K$, as follows: 


$$
g_{d}=\gamma+\varepsilon\left(u-u_{n}\right),
$$

where $\gamma$ denotes a constant term capturing the expected rate of growth; $\varepsilon$, a positive parameter; and $u_{n}$, a normal rate of capacity utilization. Firms determine the normal rate of capacity utilization through convention, historical experience, and strategic considerations. Equation (3) states that investment responds to the gap between the actual rate of capacity utilization and the normal rate of capacity utilization. ${ }^{9}$ If the actual rate of capacity utilization is equal to the normal rate of capacity utilization, firms expand plants at the same pace as the expected rate of growth. If, however, the actual rate of capacity utilization falls short of the normal rate of capacity utilization, firms consider themselves as facing excess capacity, and they decrease the rate of capital accumulation. If, on the other hand, the actual rate of capacity utilization exceeds the normal rate of capacity utilization, firms increase the rate of capital accumulation faster than the expected rate of growth.

\subsection{Short-run equilibrium}

In the short run, quantity adjustment through the rate of capacity utilization prevails. Here, $K$, $N, a$, the price $p$, and the money wage $w$ are fixed, and employment $E$ is adjusted. An equation of motion for the rate of capacity utilization can be formulated by

$$
\dot{u}=\alpha\left(g_{d}-g_{s}\right), \quad \alpha>0,
$$

where $\alpha$ denotes the speed of adjustment of the goods market. Equation (4) shows that excess demand leads to a rise in the rate of capacity utilization, while excess supply leads to a decline in the rate of capacity utilization.

The short-run equilibrium is defined as a situation where $\dot{u}=0$. In this case, we have $g_{d}=g_{s}$, which yields

$$
u^{*}=\frac{\gamma-\varepsilon u_{n}}{s m-\varepsilon} .
$$

Hereafter, short-run equilibrium values are denoted with “*." Substituting equation (5) in $g=s m u$, we obtain the short-run equilibrium rate of capital accumulation:

$$
g^{*}=\frac{s\left(\gamma-\varepsilon u_{n}\right) m}{s m-\varepsilon} .
$$

A necessary and sufficient condition for the short-run equilibrium to be stable is given by $d \dot{u} / d u<0$, which implies $s m-\varepsilon>0$. This condition is called the Keynesian stability 
condition (Marglin and Bhaduri, 1990), which means that saving responds to the rate of capacity utilization more strongly than investment. In addition, for $u^{*}>0$, we need $\gamma-\varepsilon u_{n}>0 .^{10}$ This condition means that the rate of capital accumulation is positive even when the rate of capacity utilization is zero.

The derivatives of equations (5) and (6) with respect to $s$ are both negative. That is, a rise in capitalists' propensity to save lowers both the rate of capacity utilization and the rate of capital accumulation. The latter effect is called the paradox of thrift.

The derivatives of equations (5) and (6) with respect to $m$ are both negative. That is, a rise in the profit share lowers both the rate of capacity utilization and the rate of capital accumulation. The former effect shows that the short-run equilibrium is the stagnationist regime and the latter effect shows that the short-run equilibrium is the wage-led growth regime. $^{11}$

\section{Medium-run analysis}

3.1 Dynamics of the profit share and the employment rate

In the medium run, the short-run equilibrium is always attained and the profit share and the rate of employment are adjusted. Here, $K, N, a, p$, and $w$, which are fixed in the short run, change.

From the definition of the profit share, we have $m=1-(w E / p Y)$, from which we obtain the following relationship: ${ }^{12}$

$$
\frac{\dot{m}}{1-m}=\frac{\dot{p}}{p}-\frac{\dot{w}}{w}+\frac{\dot{a}}{a}
$$

To know the dynamics of the profit share, we have to specify the dynamics of the price, the money wage, and the labor productivity.

We specify the dynamics of the money wage and price by using the theory of conflicting-claims inflation. First, suppose that the growth rate of the money wage that workers manage to negotiate depends on the discrepancy between their target profit share and the actual profit share. Second, suppose that firms set their price to close the gap between their target profit share and the actual profit share. From these considerations, the dynamics of the money wage and price can be described, respectively, as follows:

$$
\frac{\dot{w}}{w}=\theta_{w}\left(m-m_{w}\right), \quad \theta_{w}>0, \quad 0<m_{w}<1,
$$




$$
\frac{\dot{p}}{p}=\theta_{f}\left(m_{f}-m\right), \quad \theta_{f}>0, \quad 0<m_{f}<1,
$$

where $\theta_{w}$ and $\theta_{f}$ are the speed of adjustment, $m_{w}$ is the target profit share set by workers, and $m_{f}$ is the target profit share set by firms. In our model, the target profit shares are exogenously given. However, we can endogenize them. For example, Dutt (1992) and Cassetti $(2002,2003)$ assume that the target of workers depends negatively on the rate of employment. In this case, combining equation (8) with equation (9), we obtain a real-wage Phillips curve such that the growth rate of the real wage rate depends positively on the rate of employment. With this Phillips curve, we can build a Kaleckian model in which the equilibrium rate of employment is endogenously determined. ${ }^{13}$ As stated in Introduction, we use a different way to endogenize the equilibrium rate of employment.

In the following analysis, we assume that $m_{f}>m_{w}$. Firms attempt to set their targets as high as possible whereas workers attempt to set their targets as low as possible. Therefore, the assumption $m_{f}>m_{w}$ is reasonable. We can interpret $\theta_{w}$ and $\theta_{f}$ as the bargaining power of workers and that of firms, respectively (Lavoie, 1992, p. 393; Cassetti, 2002, p. 192; Cassetti, 2003, p. 453). We assume $\theta_{f}+\theta_{w}=1$ and define $\theta_{f} \equiv \theta$ because bargaining power is a relative concept. We then obtain $\theta_{w}=1-\theta$, where $0<\theta<1 .^{14}$ For example, we can consider an increase in the unionization rate as a factor for raising the bargaining power of workers (i.e., a decrease in $\theta$ ), and an increase in the market power of oligopolistic firms as a factor for raising the bargaining power of firms (i.e., an increase in $\theta$ ).

We now turn to the specification of endogenous technological change. As stated above, we assume that the growth rate of labor productivity $g_{a}=\dot{a} / a$ depends positively on the rate of employment $e$.

$$
g_{a}(e)=\lambda e^{\psi}, \quad \lambda>0, \psi>0,
$$

where $e=E / N$ denotes the rate of employment, $N$ the exogenous labor supply, $\lambda$ a positive constant, and $\psi$ the elasticity of the growth rate of labor productivity with respect to the rate of employment. We use the above specification to conduct numerical simulations in what follows. Note, however, that our results do not depend on this specification as long as the growth rate of labor productivity is increasing in the rate of employment. In addition, the non-linearity of equation (10), when $\psi \neq 1$, does not concern with the local stability of equilibrium and results of comparative statics analysis. Nevertheless, the non-linearity can 
affect the global behavior of each endogenous variable.

Let us explain the difference between equation (10) and specifications of Bhaduri (2006) and Dutt (2006). Equation (10) and their specifications bear similarities but differ in some respects. In Bhaduri's (2006) model, a change in the growth rate of labor productivity $\left(\dot{g}_{a}\right)$ depends positively on the rate of change in the employment rate $(\dot{e} / e)$. Dutt (2006) presents two kinds of specifications. In the first model of Dutt (2006), the rate of change in the growth rate of labor productivity $\left(\dot{g}_{a} / g_{a}\right)$ depends positively on the rate of change in the employment rate $(\dot{e} / e)$. In these specifications, it is the growth rate of labor productivity, not the employment rate that is endogenously determined. In this paper, to examine how the employment is determined, we relate the growth rate of labor productivity $\left(g_{a}\right)$ to the employment rate $(e)$. In the second model of Dutt (2006), the rate of change in the growth rate of labor productivity $\left(\dot{g}_{a} / g_{a}\right)$ depends negatively on the employment rate $(e)$. In addition, when the employment rate is equal to its natural rate $e_{N}$, the rate of change in the growth rate of labor productivity will be zero. From this, the steady-state employment rate is equal to the natural rate. The natural rate $e_{N}$ is exogenously given, and then, it does not depend on other parameters of the model. In our model, both the growth rate of labor productivity and the employment rate are simultaneously determined, and moreover, the employment rate does depend on parameters.

Substituting equations (8), (9), and (10) in equation (7), we obtain an equation of motion for the profit share:

$$
\dot{m}=-(1-m)\left[m-A-g_{a}(e)\right],
$$

where $A \equiv \theta m_{f}+(1-\theta) m_{w}$. We assume that $A>n$, which means that a weighted average of the two groups' target profit shares is larger than the growth rate of labor supply. Given that the size of $n$ is about 10 percent at most, this assumption is plausible.

Let us derive an equation of motion for the rate of employment. From equation (2), the rate of employment rate is given by $e=u K /(a N)$, from which the rate of change of $e$ leads to

$$
\frac{\dot{e}}{e}=\frac{\dot{u}}{u}+g_{d}-g_{a}(e)-n,
$$

where $n$ is the growth rate of $N$ and given exogenously.

3.2 Medium-run equilibrium 
In the medium run, as stated above, the short-run equilibrium is always attained, and consequently, the rate of change of $u$ is zero. Substituting equations (3) and (10) in equation (12), we obtain the following expression:

$$
\dot{e}=e\left[\frac{s\left(\gamma-\varepsilon u_{n}\right) m}{s m-\varepsilon}-g_{a}(e)-n\right] .
$$

Equations (11) and (13) describe the medium-run dynamics.

Let us find the medium-run equilibrium. Combining $\dot{m}=0$ with $\dot{e}=0$, we obtain the following quadratic equation for the profit share:

$$
s m^{2}-\Theta m+\varepsilon(A-n)=0,
$$

where $\Theta \equiv s\left(\gamma-\varepsilon u_{n}\right)+s(A-n)+\varepsilon>0$.

From equation (14), we obtain two real and distinct roots. However, the larger root corresponds to the medium-run equilibrium value. ${ }^{15}$

$$
m^{* *}=\frac{\Theta+\sqrt{\Theta^{2}-4 s \varepsilon(A-n)}}{2 s} .
$$

Hereafter, medium-run equilibrium values are denoted with “**." Using equation (15), we obtain the rate of employment in the medium-run equilibrium.

$$
e^{* *}=\left(\frac{m^{* *}-A}{\lambda}\right)^{1 / \psi} \text {. }
$$

To analyze the local stability of the medium-run equilibrium, we linearize equations (11) and (13) around the equilibrium. The elements of the Jacobian matrix $\mathbf{J}$ are given by

$$
\begin{aligned}
& J_{11}=\frac{\partial \dot{m}}{\partial m}=-(1-m)<0, \\
& J_{12}=\frac{\partial \dot{m}}{\partial e}=(1-m) g_{a}^{\prime}(e)>0, \\
& J_{21}=\frac{\partial \dot{e}}{\partial m}=-\frac{e s \varepsilon\left(\gamma-\varepsilon u_{n}\right)}{(s m-\varepsilon)^{2}}<0, \\
& J_{22}=\frac{\partial \dot{e}}{\partial e}=-e g_{a}^{\prime}(e)<0 .
\end{aligned}
$$

All elements are evaluated at the medium-run equilibrium, though we omit “**" to avoid troublesome notations. Necessary and sufficient conditions for the stability of the medium-run equilibrium are given by both $\operatorname{tr} \mathbf{J}<0$ and $\operatorname{det} \mathbf{J}>0$, where $\operatorname{tr} \mathbf{J}$ denotes the trace of $\mathbf{J}$ 
and $\operatorname{det} \mathbf{J}$ the determinant of $\mathbf{J}$. In our model, these conditions are satisfied because

$$
\begin{aligned}
& \operatorname{tr} \mathbf{J}=J_{11}+J_{22}=(-)+(-)<0, \\
& \operatorname{det} \mathbf{J}=J_{11} J_{22}-J_{12} J_{21}=(-)(-)-(+)(-)>0 .
\end{aligned}
$$

Therefore, the medium-run equilibrium is locally stable.

\subsection{Comparative statics in the medium-run equilibrium}

Table 1 summarizes the results of comparative statics in the medium-run equilibrium. ${ }^{16}$

Table 1: Results for medium-run comparative statics analysis

\begin{tabular}{cccc} 
& $A$ & $s$ & $n$ \\
\hline$m^{* *}$ & + & - & - \\
\hline$e^{* *}$ & - & - & - \\
\hline$u^{* *}$ & - & - & + \\
\hline$g^{* *}$ & - & - & + \\
\hline Note: $A \equiv \theta m_{f}+(1-\theta) m_{w}$ and $m_{f}>m_{w}$.
\end{tabular}

Note that an increase in $A$ means an increase in $\theta \cdot{ }^{17}$ An increase in the bargaining power of firms decreases the rate of utilization rate and the rate of employment. This implies that a policy for weakening the bargaining power of workers cannot lower the rate of unemployment. Stockhammer (2004) also investigates the relationship between bargaining power and unemployment. He concludes that in the profit-led growth regime, a decline in the bargaining power of workers leads to lower unemployment. However, in the wage-led growth regime of Stockhammer's model, the equilibrium is unstable, and consequently, we cannot investigate the relationship between bargaining power and unemployment. ${ }^{18}$ In our model, in contrast, the medium-run equilibrium is stable even though it is wage-led growth. The reason for this stability is a stabilizing effect of the endogenous labor-saving technological change. Our result is consistent with the empirical result of Storm and Naastepad (2007, 2008). Using data for 20 OECD countries during the period 1984-1997, they show that an increase in the bargaining power of firms due to labor market deregulation raises the rate of unemployment in contrast to the view of mainstream theory. 
An increase in the saving rate decreases the rate of capacity utilization and the rate of employment. In Stockhammer (2004), the equilibrium rate of employment consists of the exogenous natural rate of growth and parameters of the investment function and the income distribution function, and does not depend on the saving rate. Hence, a change in the saving rate never affects the rate of employment. In our model, on the other hand, the natural rate of growth is endogenously determined and a change in the saving rate affects the rate of employment accordingly.

The effect of an increase in the target profit shares $m_{f}$ and $m_{w}$ is similar to that of an increase in $\theta$ discussed above. An important issue in the Kaleckian tradition is what regime is obtained in the equilibrium. As stated in the short-run analysis, classification of regimes is based on the relationship between the profit share, the rate of capacity utilization and the rate of capital accumulation. Note, however, that the profit share in the medium-run is not an exogenous but an endogenous variable. Nevertheless, because $m_{f}$ and $m_{w}$ change in the same direction as $m^{* *}$, we can use these variables as a proxy for the actual profit share. An increase in $m_{f}$ or $m_{w}$ leads to a decline in both $u^{* *}$ and $g^{* *}$. From this, we can conclude that the medium-run equilibrium corresponds to both stagnationist regime and wage-led growth regime, which are typical of the Kaleckian model.

\section{Long-run analysis}

4.1 Dynamics of the normal rate of capacity utilization and the expected rate of growth In the long run, the medium-run equilibrium is always attained and the normal rate of capacity utilization and the expected rate of growth are adjusted. We follow Lavoie (1996), Dutt (1997), Lavoie et al. (2004), and Cassetti (2006) to describe the long-run dynamics. ${ }^{19}$

First, we introduce the following adjustment equation for the normal rate of capacity utilization:

$$
\dot{u}_{n}=\phi\left(u^{* *}-u_{n}\right), \quad \phi>0,
$$

where $\phi$ denotes the speed of adjustment. Equation (17) states that the normal rate of capacity utilization is adjusted according to the gap between the actual rate of capacity utilization and the normal rate of capacity utilization. Note that we have $u^{* *}=u^{* *}\left(u_{n}, \gamma\right)$ from the medium-run analysis.

Second, we introduce the following adjustment equation for the expected rate of capital 
accumulation:

$$
\dot{\gamma}=\eta\left(g^{* *}-\gamma\right), \quad \eta>0,
$$

where $\eta$ denotes the speed of adjustment. Equation (18) shows that the expected rate of growth is adjusted according to the gap between the actual growth rate and the expected rate of growth, which corresponds to adaptive expectations. Because we have $g^{* *}=\gamma+\varepsilon\left(u^{* *}-u_{n}\right)$, we can rewrite equation (18) as follows:

$$
\dot{\gamma}=\eta \varepsilon\left(u^{* *}-u_{n}\right) .
$$

The empirical validity of simultaneous adjustments of $u_{n}$ and $\gamma$ is discussed by Lavoie et al. (2004). They empirically test four kinds of investment functions - the French Marxist equation, the naive Kaleckian equation, the American Marxist equation, and the hysteresis Kaleckian equation-by using data from the Canadian manufacturing sector and the total industrial sector during the period 1960-2000. Their results show that the hysteresis Kaleckian equation performs better than the other three specifications. In contrast, Skott (2008) criticizes Lavoie et al. (2004): their estimated empirical hysteresis Kaleckian equation bears no relation to the theoretical model. In either case, because we cannot directly observe the normal rate of capacity utilization, we need to devise methods for an empirical study. ${ }^{20}$ In addition, because Lavoie et al. (2004) and Skott (2008) use only Canadian data, we also need to investigate other countries to ascertain the validity of the hysteresis Kaleckian investment function.

\subsection{Long-run equilibrium}

We derive the long-run equilibrium. A system of differential equations composed of equations (17) and (19) takes a special form called a zero root system, in which one eigenvalue of the Jacobian matrix is zero. ${ }^{21}$

In the long-run equilibrium, we obtain $u^{* *}=u_{n}$, which yields the following relationship between $u_{n}$ and $\gamma::^{22}$

$$
\gamma=\frac{s(A-n) u_{n}}{1-s u_{n}}
$$

This is an upward-sloping curve through the origin with $u_{n}=1 / s$ being an asymptote. Points on this curve correspond to the long-run equilibria. Note, however, that not all the points on this curve are long-run equilibria and some constraints are imposed. To begin with, the rate of 
capacity utilization has to be smaller than unity. This constraint is located to the left of the asymptote $u_{n}=1 / s$ because $0<s<1$. Next, we have $\gamma>\varepsilon u_{n}$ from the short-run analysis. This constraint means that the long-run equilibria have to be located above the straight line $\gamma=\varepsilon u_{n}$. Finally, we have $s m-\varepsilon>0$ from the short-run analysis. This constraint means that the long-run equilibria have to be larger than $\gamma=[\varepsilon-s(A-n)] / s$.

For any initial condition within the constraints, there exists a unique long-run equilibrium. ${ }^{23}$ From equations (17) and (19), we obtain the relationship $\dot{\gamma}=(\eta \varepsilon / \phi) \dot{u}_{n}$, from which we have the constant ratio $\gamma=(\eta \varepsilon / \phi) u_{n}$ along the transitional process. Hence, the transitional process is given by the following upward sloping straight line:

$$
\gamma(t)=\frac{\eta \varepsilon}{\phi} u_{n}(t)+\gamma(0)-\frac{\eta \varepsilon}{\phi} u_{n}(0)
$$

where $\gamma(0)$ and $u_{n}(0)$ are initial conditions. The intersection of equations (21) and (20) yields the long-run equilibrium.

The long-run equilibrium values are given by

$$
\begin{aligned}
g^{* * *} & =\gamma^{* * *}, \\
g_{a}^{* * *} & =\gamma^{* * *}-n, \\
m^{* * *} & =\gamma^{* * *}+A-n, \\
u^{* * *} & =\frac{\gamma^{* * *}}{s\left(\gamma^{* * *}+A-n\right)}, \\
e^{* * *} & =\left(\frac{\gamma^{* * *}-n}{\lambda}\right)^{1 / \psi} .
\end{aligned}
$$

The long-run equilibrium values are denoted with “***." Given initial conditions, we can determine the long-run value of $\gamma^{* * *}$, which determines equations (22) through (26). What is important here is that different initial conditions produce different long-run values of $\gamma^{* * *}$. Therefore, the long-run equilibrium shows path-dependency.

Figure 1: Convergence to the long-run equilibrium

Figure 1 shows the long-run phase diagram. As stated above, the locus of $\dot{\gamma}=\dot{u}_{n}=0$ is an upward-sloping curve. The constraints $\gamma=\varepsilon u_{n}$ and $\gamma=[\varepsilon-s(A-n)] / s$ are also drawn in 
Figure $1 .^{24}$ The solution path denoted by the solid line starting from point A converges to the long-run equilibrium point denoted by $\mathrm{E}_{1}$, whereas the solution path denoted by the solid line starting from point $\mathrm{B}$ converges to long-run equilibrium $\mathrm{E}_{2}$. For this reason, if the initial point is different, then the corresponding long-run equilibrium is also different.

The long-run equilibrium is not always stable. If the coefficient $\eta \varepsilon / \phi$, the slope of the solution path, is extremely large, the economy cannot reach the long-run equilibrium. This phenomenon arises when the speed of adjustment of the expected rate of growth is large, when the coefficient of the investment function is large, and when the speed of adjustment of the rate of capacity utilization is small. These discussions are also shown in Figure 1. The solution path denoted by the broken line starting from point A crosses the locus of $\dot{\gamma}=\dot{u}_{n}=0$ at a point where $u_{n}$ exceeds unity, and consequently, the path is divergent. The solution path denoted by the broken line starting from point B crosses the constraint, and consequently, that path is also divergent.

Moreover, even if the slope of the solution path is small, the economy cannot reach the long-run equilibrium depending on the position of the initial value.

From these observations, it follows that both the size of the speed of adjustment and the initial position are crucial to the stable convergence to the long-run equilibrium.

\subsection{Comparative statics in the long-run equilibrium}

Table 2 shows the results of comparative statics in the long-run equilibrium.

Table 2: Results for long-run comparative statics analysis

\begin{tabular}{cccc} 
& $A$ & $s$ & $n$ \\
\hline$m^{* * *}$ & + & - & - \\
\hline$e^{* * *}$ & - & - & - \\
\hline$u^{* * *}$ & - & - & + \\
\hline$g^{* * *}$ & - & - & + \\
\hline
\end{tabular}

When $s$ or $A$ increases, the curve represented by equation (20) rotates counterclockwise around the origin. Because the solution path is unaffected by the change in $s$ or $A$, the 
intersection of the solution path and the curve moves toward the lower left. Therefore, both the normal rate of capacity utilization and the expected rate of growth decrease.

When $n$ increases, the curve rotates clockwise, and hence, the long-run equilibrium values of $u^{* * *}$ and $\gamma^{* * *}$ increase.

The results in Table 2 are the same as those in Table 1. In the long run, an increase in the bargaining power of firms also lowers the rate of employment.

Let us compare our results with those of Dutt (2006). In the long run of Dutt's model, the growth rate of labor productivity and the autonomous rate of growth (i.e., the expected rate of growth in our model) are adjusted. The adjustment of labor productivity growth is equivalent to the adjustment of the rate of employment because in Dutt's model, productivity growth is an increasing function of the rate of employment. The long-run equilibrium has a path-dependent property and depends on the saving rate, the growth rate of labor supply, and the coefficient of the rate of capacity utilization in the investment function. An increase in the saving rate lowers the rate of employment and the rate of capital accumulation. An increase in the growth rate of labor supply lowers the rate of employment and raises the rate of capital accumulation. These results are the same as our results. A rise in the coefficient of the rate of capacity utilization in the investment function increases both the rate of employment and the rate of capital accumulation. In our model, in contrast, a change in the coefficient in the investment function does not affect the long-run equilibrium value: in the long-run equilibrium, the actual rate of capacity utilization and the normal rate of capacity utilization are equalized, and accordingly, the effect of the rate of utilization on investment vanishes. Our model extends the results of Dutt (2006) in that (i) unlike Dutt's model, long-run income distribution is endogenously determined and (ii) our model can investigate the effect of the bargaining power on the rate of employment.

How are our results modified if we do not consider the adjustment process of the expected rate of growth and if we consider only the adjustment process of the normal rate of capacity utilization? This corresponds to considering $\gamma$ as a parameter rather than an endogenous variable. In this case, the adjustment process of $u_{n}$ is stable and the long-run equilibrium values are given by equations (22) through (26) with $\gamma$ being a parameter. It follows from this that the long-run equilibrium values of the rate of capital accumulation, the growth rate of labor productivity, and the rate of employment do not depend on relative bargaining power. 
On the other hand, how are our results modified if we do not consider the adjustment process of the normal rate of capacity utilization and if we consider only the adjustment process of the expected rate of growth? In this case, the adjustment process of $\gamma$ is unstable: if there is any divergence from the long-run equilibrium, then the divergence continues to expand cumulatively. This reminds us of Harrod's instability principle (Lavoie, 1995, p. 806).

Summarizing these discussions, we can understand that because we allow both the normal rate of capacity utilization and the expected rate of growth to adjust simultaneously, the long-run equilibrium will be stable and depend on bargaining power.

The adjustment process of the expected rate of growth also concerns the long-run equilibrium regime. As is the case with the medium-run equilibrium, we investigate the relationship between $m_{f}$ or $m_{w}$, and $u^{* * *}$ and $g^{* * *}$. An increase in $m_{f}$ or $m_{w}$ lowers the long-run equilibrium value of the rate of capacity utilization, irrespective of whether the expected rate of growth is endogenous or exogenous. This means that the long-run equilibrium is the stagnationist regime. If the expected rate of growth is endogenous, then an increase in $m_{f}$ or $m_{w}$ lowers the long-run equilibrium value of the rate of capital accumulation, which shows that that the long-run equilibrium is the wage-led growth regime. However, if the expected rate of growth is exogenous, then changes in $m_{f}$ and $m_{w}$ do not affect the long-run equilibrium value of the rate of capital accumulation.

\subsection{Numerical examples}

In the foregoing analysis, we separate the short run, the medium run, and long run. In this subsection, we consider the adjustment process of the five endogenous variables all together: the rate of capacity utilization, profit share, employment rate, normal rate of capacity utilization, and expected rate of growth. The property of the equilibrium is identical in both the model in which the three runs are distinguished and the five-variable model. However, the transitional dynamics toward equilibrium can differ between the two models. For this purpose, we need to analyze the system of five differential equations that consist of equations (4), (11), (12), (17), and (18). Whereas analytical treatment of high-order differential equations is troublesome, numerical analysis is relatively easy, which we now turn to. ${ }^{25}$

For numerical simulations, we set parameters as follows: 


$$
\theta=0.3, m_{f}=0.3, m_{w}=0.2, s=0.75, n=0.05, \varepsilon=0.08, \alpha=1, \lambda=0.15, \psi=0.9, \phi=1, \eta=1 .
$$

If the five-dimensional model exhibits path-dependency, different initial conditions must produce different steady-state values under exactly the same parameter setting. Table 3 shows the results of this numerical analysis.

Table 3: Results of numerical simulations for the five-dimensional dynamical system

\begin{tabular}{ccccc} 
& $\begin{array}{l}\text { Initial values: } \\
\text { Case 1 }\end{array}$ & $\begin{array}{l}\text { Long-run } \\
\text { equilibrium: } \\
\text { Case 1 }\end{array}$ & $\begin{array}{l}\text { Initial values: } \\
\text { Case 2 }\end{array}$ & $\begin{array}{l}\text { Long-run } \\
\text { equilibrium: } \\
\text { Case 2 }\end{array}$ \\
\hline$u$ & 0.6 & 0.39 & 0.7 & 0.69 \\
$m$ & 0.2 & 0.26 & 0.3 & 0.37 \\
$e$ & 0.7 & 0.14 & 0.8 & 0.93 \\
$u_{n}$ & 0.7 & 0.39 & 0.8 & 0.69 \\
$\gamma$ & 0.1 & 0.08 & 0.2 & 0.19 \\
\hline \hline
\end{tabular}

The initial values in Case 1 are smaller than those in Case 2. From Table 3, we find that if we change initial conditions, we obtain different long-run equilibrium values. Therefore, in this five-dimensional dynamical system, we also have path-dependency. ${ }^{26}$

Moreover, non-monotonic dynamics emerge in the five-dimensional system. Parameters and initial values are set as follows:

$$
\begin{gathered}
\theta=0.3, m_{f}=0.3, m_{w}=0.2, s=0.7, n=0.05, \varepsilon=0.3, \alpha=7, \lambda=0.15, \psi=1.2, \phi=1, \eta=1 \\
u(0)=0.6, m(0)=0.3, e(0)=0.8, u_{n}(0)=0.8, \gamma(0)=0.2 .
\end{gathered}
$$

Note that in this case, $\varepsilon$, that is, the sensitivity of investment to the capacity utilization, and $\alpha$, that is, the adjustment speed of the goods market, are larger than those in Cases 1 and 2. Larger $\varepsilon$ and $\alpha$ are destabilizing factors. Time path of each variable is drawn in Figure 2, from which we can see that each variable converge to its long-run equilibrium with oscillation. Whereas we do not observe such a behavior when we separate the three runs, we can observe a cyclical fluctuation when we combine the three runs altogether.

We note that in the five-dimensional system, $u_{n}$ and $\gamma$ synchronize as in the model in section 4. Hence, if we plot the dynamics of $u_{n}$ and $\gamma$ on the $u_{n}-\gamma$ plane, we obtain a 
solution path as one in figure 1.

Figure 2: Cyclical fluctuations in the five-dimensional system

\section{Conclusion}

In this paper, we have developed a Kaleckian model that considers the determination of the rate of employment. For this purpose, we have introduced endogenous technological change into a Kaleckian model with conflicting-claims inflation.

We have also discussed how the endogenous variables in our model are determined in the long run where the normal rate of capacity utilization and the expected rate of growth are adjusted. This is an answer to the criticism that the Kaleckian model lacks logical consistency in the long run. We have shown that the results in the medium-run equilibrium are carried over to the long-run equilibrium. In this sense, the Kaleckian model can be used for long-run analysis.

In our model, both the medium-run and the long-run employment rate depend on the relative bargaining power between workers and capitalists. A rise in the bargaining power of capitalists increases the unemployment rate, which is contrary to the assertion of mainstream theory. As long as the medium-run and the long-run regime experience wage-led growth, a policy intended to raise the bargaining power of capitalists is unfavorable for a reduction in the unemployment rate.

Long-run equilibrium values have path-dependent properties. As such, slightly different initial conditions are likely to produce large differences in both the transitional path to the long-run equilibrium and the position of the long-run equilibrium itself, which can account for cross-country differences in the long-run unemployment rate.

\section{References}

Agliardi, E. 1988 Microeconomic foundations of macroeconomics in the post-Keynesian approach. Metroeconomica 39, no. 3: 275-297.

Amadeo, E. 1986. Notes on capacity utilisation, distribution and accumulation. Contributions to Political Economy 5, no. 1: 83-94.

Auerbach, P., and P. Skott. 1988. Concentration, competition and distribution-a critique of 
theories of monopoly capital. International Review of Applied Economics 2, no. 1: 42-61.

Bhaduri, A., and S. Marglin. 1990. Unemployment and the real wage: the economic basis for contesting political ideologies. Cambridge Journal of Economics 14, no. 4: 375-393.

Bhaduri, A. 2006. Endogenous economic growth: a new approach. Cambridge Journal of Economics 30, no. 1: 69-83.

Blecker, R.A. 2002. Distribution, demand and growth in neo-Kaleckian macro-models. In The Economics of Demand-led Growth, Challenging the Supply-side Vision of the Long Run, ed. M. Setterfield. Cheltenham: Edward Elgar.

Cassetti, M. 2002. Conflict, inflation, distribution and terms of trade in the Kaleckian model. In The Economics of Demand-led Growth, Challenging the Supply-side Vision of the Long Run, ed. M. Setterfield. Cheltenham: Edward Elgar.

Cassetti, M. 2003. Bargaining power, effective demand and technical progress: a Kaleckian model of growth. Cambridge Journal of Economics 27, no. 3: 449-464.

Cassetti, M. 2006. A note on the long-run behaviour of Kaleckian models. Review of Political Economy 18, no. 4: 497-508.

Duménil, G., and D. Lévy. 1999. Being Keynesian in the short term and classical in the long term: the traverse to classical long-term equilibrium. The Manchester School 67, no. 6: 684-716.

Dutt, A.K. 1987. Alternative closures again: a comment on Growth, distribution and inflation. Cambridge Journal of Economics 11, no. 1: 75-82.

Dutt, A.K. 1992. Conflict inflation, distribution, cyclical accumulation and crises. European Journal of Political Economy 8, no. 4: 579-597.

Dutt, A.K., and E.J. Amadeo. 1993. A post-Keynesian theory of growth, interest and money. In The Dynamics of the Wealth of Nations: Growth, Distribution and Structural Change, ed. M. Baranzini and G.C. Harcourt. New York: St. Martin’s Press.

Dutt, A.K. 1997. Equilibrium, path dependence and hysteresis in Post-Keynesian models. In Markets, Unemployment and Economic Policy: Essays in Honour of Geoff Harcourt, Vol. 2, ed. P. Arestis, G. Palma, and M. Sawyer. London: Routledge.

Dutt, A.K. 2006. Aggregate demand, aggregate supply and economic growth. International Review of Applied Economics 20, no. 3: 319-336.

Foley, D.K., and T.R. Michl. 1999. Growth and Distribution. Cambridge, MA: Harvard 


\section{University Press.}

Giavazzi, F., and C. Wyplosz. 1985. The zero root problem: a note on the dynamic determination of the stationary equilibrium in linear models. Review of Economic Studies 52, no. 2: 353-357.

Kalecki, M. 1971. Selected Essays on the Dynamics of the Capitalist Economy. Cambridge: Cambridge University Press.

van de Klundert, T., and T. van Schaik. 1990. Unemployment persistence and loss of productive capacity: a Keynesian approach. Journal of Macroeconomics 12, no. 3: 363-380.

Lavoie, M. 1992. Foundations of Post-Keynesian Economic Analysis. Cheltenham: Edward Elgar.

Lavoie, M. 1995. The Kaleckian model of growth and distribution and its neo-Ricardian and neo-Marxian critiques. Cambridge Journal of Economics 19, no. 6: 789-818.

Lavoie, H. 1996. Traverse, hysteresis and normal rates of capacity utilization in Kaleckian models of growth and distribution. Review of Radical and Political Economics 28, no. 4: 113-147.

Lavoie, M. 2002. The Kaleckian growth model with target return pricing and conflict inflation. In The Economics of Demand-led Growth, Challenging the Supply-side Vision of the Long Run, ed. M. Setterfield. Cheltenham: Edward Elgar.

Lavoie, M. 2003. Kaleckian effective demand and Sraffian normal prices: towards a reconciliation. Review of Political Economy 15, no. 1: 53-74.

Lavoie, M. 2006. Introduction to Post-Keynesian Economics. New York: Palgrave Macmillan.

Lavoie, M., G. Rodríguez, and M. Seccareccia. 2004. Similitudes and discrepancies in Post-Keynesian and Marxist theories of investment: a theoretical and empirical investigation. International Review of Applied Economics 18, no. 2: 127-149.

Léon-Ledesma, M.A., and A.P. Thirlwall. 2002. The endogeneity of the natural rate of growth. Cambridge Journal of Economics 26, no. 4: 441-459.

Libânio, G.A. 2009. Aggregate demand and the endogeneity of the natural rate of growth: evidence from Latin American economies. Cambridge Journal of Economics 33, no. 5: 967-984.

Lima, G.T. 2004. Endogenous technological innovation, capital accumulation and 
distributional dynamics. Metroeconomica 55, no. 4: 386-408.

Marglin, S., and A. Bhaduri. 1990. Profit squeeze and Keynesian theory. In The Golden Age of Capitalism: Reinterpreting the Postwar Experience, ed. S. Marglin and J. Schor. Oxford: Clarendon Press.

Marquetti, A. 2004. Do rising real wages increase the rate of labor-saving technical change? some econometric evidence. Metroeconomica 55, no. 4: 432-441.

Mott, T., and E. Slattery. 1994. The influence of changes in income distribution on aggregate demand in a Kaleckian model: stagnation vs. exhilaration reconsidered. In Employment, Growth and Finance. ed. P. Davidson and J.A. Kregel. Cheltenham: Edward Elgar.

Ohno, T. 2009. Post-Keynesian effective demand and capital-labour substitution. Metroeconomica 60, no. 3: 525-536.

Park, M.S. 1997. Normal values and average values. Metroeconomica 48, no. 2: 188-199.

Rowthorn, R.E. 1977. Conflict, inflation and money. Cambridge Journal of Economics 1, no. 3: $215-239$.

Rowthorn, R.E. 1981. Demand, real wages and economic growth. Thames Papers in Political Economy, Autumn: 1-39.

Skott, P. 2008. Theoretical and empirical shortcomings of the Kaleckian investment function. Department of Economics Working Paper 2008-11, University of Massachusetts Amherst.

Stockhammer, E. 2004. Is there an equilibrium rate of unemployment in the long run? Review of Political Economy 16, no. 1: 59-77.

Stockhammer, E., and O. Onaran. 2004. Accumulation, distribution and employment: a structural VAR approach to a Kaleckian macro model. Structural Change and Economic Dynamics 15, no. 4: 421-447.

Storm, S., and C.W.M. Naastepad. 2007. It is high time to ditch the NAIRU. Journal of Post Keynesian Economics 29, no. 4: 531-554.

Storm, S., and C.W.M. Naastepad. 2008. The NAIRU reconsidered: why labour market deregulation may raise unemployment. International Review of Applied Economics 22, no. 5: 527-544.

Taylor, L. 2004. Reconstructing Macroeconomics: Structuralist Proposals and Critiques of the Mainstream. Cambridge, MA: Harvard University Press.

Vogel, L. 2009. The endogeneity of the natural rate of growth-an empirical study for 
Latin-American countries. International Review of Applied Economics 23, no. 1: 41-53. You, J.-I. 1994. Macroeconomic structure, endogenous technical change and growth. Cambridge Journal of Economics 18, no. 2: 213-233.

\section{Notes}

1 See Kalecki (1971) for his economic theory. For the framework of the Kaleckian model, see Rowthorn (1981), Lavoie (1992), Foley and Michl (1999, ch. 10), Blecker (2002), and Taylor (2004, ch. 5).

2 The theory of conflicting-claims inflation is developed by Rowthorn (1977). For Kaleckian models with the conflicting-claims inflation, see Dutt (1987) and Cassetti (2002, 2003, 2006).

${ }^{3}$ Ohno (2009) presents a long-run Kaleckian model which considers capital-labor substitution and increasing returns to scale in the production function. In the long-run equilibrium, the desired capital-labor ratio and the actual capital-labor ratio are equalized.

${ }^{4}$ Cassetti (2002, pp. 205-206) also points out that the long-run rate of employment in the conventional Kaleckian models is not constant.

5 Rowthorn (1981), Lavoie (1992, p. 322), You (1994), Cassetti (2003), and Stockhammer and Onaran (2004) also endogenize technical progress in the Kaleckian model through use of Kaldorian technical progress functions. Lima (2004) develops a Kaleckian model with endogenous technical progress in which the growth rate of labor productivity depends non-linearly on the wage share.

${ }^{6}$ The view that increases in wages induce labor-saving technical change is consistent with an empirical study by Marquetti (2004), who investigates the co-integration between real wages and labor productivity by using U.S. data.

${ }^{7}$ When the rate of capacity utilization changes, the rate of employment necessarily changes. As such, we should combine the short run and the medium run and consider the adjustment processes of the rate of capacity utilization, the rate of employment, and the profit share all together. However, we separate the short run from the medium run because a three-dimensional system is very complicated and because the property of the equilibrium is identical in both the three-dimensional system and our system.

${ }^{8}$ Given the fixed coefficients production function, a cost minimizing firm operates at a point 
on isoquant curves such that $a E=(u / k) K$, from which we obtain $a=Y / E$.

${ }^{9}$ Cassetti (2006) uses the investment function that contains the rate of profit as an endogenous variable in addition to the rate of capacity utilization. To simplify the analysis, our model uses the investment function that contains only the rate of capacity utilization. Introducing the rate of profit as a second variable does not change the main results in this paper. For the specification of the investment function, see also Marglin and Bhaduri (1990). They assert that the profit share, not the rate of profit, should be a variable in the investment function. In this case, according to the shape of the investment function, we obtain various equilibrium regimes. For this issue, see also Bhaduri and Marglin (1990) and Blecker (2002). Agliardi (1988) and Mott and Slattery (1994) disagree with the logic of Bhaduri and Marglin (1990).

${ }^{10}$ For the rate of capacity utilization to be smaller than unity, we need $s m-\varepsilon>\gamma-\varepsilon u_{n}$ in addition to these two conditions.

11 Our classification of regimes is based on Blecker (2002). The stagnationist regime contrasts with the exhilarationist regime and the wage-led growth regime contrasts with the profit-led growth regime.

12 Cassetti $(2002,2003,2006)$ derives an equation of motion for the profit share by specifying a price-setting equation of firms and differentiating it with respect to time. However, this procedure is unnecessary for deriving the equation of motion for the profit share; plus, our procedure is easier than his procedure. With the conflicting-claims inflation theory, the price-setting equation in Cassetti's model plays the role of determining the mark-up rate rather than the price level.

13 If we introduce both the real-wage Phillips curve and equation (10) into our model, the medium-run equilibrium can be stable or unstable depending on which effect dominates, the effect of the real-wage Phillips curve or the effect of equation (10). However, as long as the medium-run equilibrium is stable, results of comparative statics analysis are the same as those of our model. Moreover, we can assume that the target profit share of workers depends negatively on the growth rate of labor productivity while the target profit share of firms depends positively on the growth rate of labor productivity. Because the growth rate of labor productivity is an increasing function of the employment rate, it amounts to say that the workers' target is a decreasing function of the employment rate while the firms' target is an 
increasing function of the employment rate. In this case also, as long as the medium-run equilibrium is stable, similar arguments hold. For details, see section A-3 of the Appendix, which is available on request.

14 The constraint $0<\theta_{w}, \theta_{f}<1$ is also adopted by Dutt and Amadeo (1993), who, however, do not assume $\theta_{f}+\theta_{w}=1$. Even if we impose only $0<\theta_{w}, \theta_{f}<1$ and not $\theta_{f}+\theta_{w}=1$, we obtain similar results.

15 For details, see section A-1 of the Appendix, which is available on request.

16 For details of comparative statics analysis, see section A-2 of the Appendix, which is available on request.

17 Recall that we assume that $m_{f}>m_{w}$. With this assumption, an increase in $A$ corresponds to an increase in $\theta$.

18 Stockhammer (2004) uses a Marglin and Bhaduri (1990) type of investment function, thereby leading to both wage-led growth and profit-led growth in the equilibrium.

19 Cassetti (2006) develops a model in which in addition to the normal rate of capacity utilization and the expected rate of capital accumulation, the normal rate of profit and the drop-out ratio of capital equipment are also adjusted in the long run.

${ }^{20}$ Lavoie et al. (2004) apply the Hodrick-Prescott filter to the actual series of capacity utilization to obtain the series of normal rates of capacity utilization. Skott (2008) uses the Koyck transformation to delete the normal rate of capacity utilization from the estimated equation, and accordingly, he dispense with unobservable variables.

21 The other eigenvalue is the trace of the Jacobian matrix.

22 For the derivation of equation (20), see section A-4 of the Appendix, which is available on request.

${ }^{23}$ For the solution method below, see Giavazzi and Wyplosz (1985) and van de Klundert and van Schaik (1990).

${ }^{24}$ Indeed, in addition to these two constraints, there are two additional constraints: one is given by $\gamma<n+\lambda$, which represents that the long-run equilibrium rate of employment is less than unity; the other is given by $\gamma<1-(A-n)$, which represents that the long-run equilibrium profit share is less than unity. For ease of presentation, we omit the two additional constraints. 
25 For numerical computation, we use Mathematica 7. The Mathematica code used is available on request.

${ }^{26}$ For the time paths for cases 1 and 2, see sections A-5 and A-6 of the Appendix, which is available on request. 


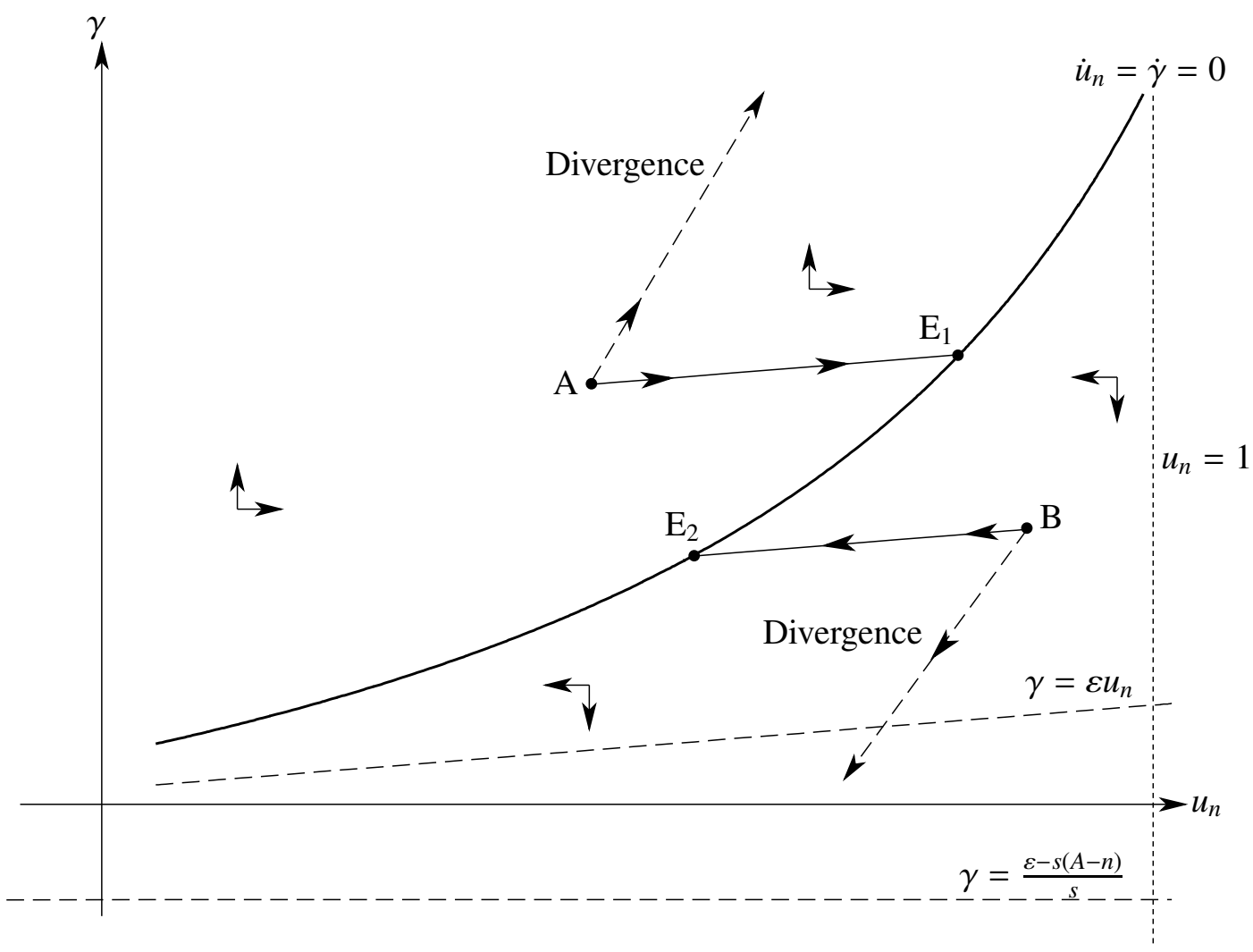

Figure 1: Convergence to the long-run equilibrium 

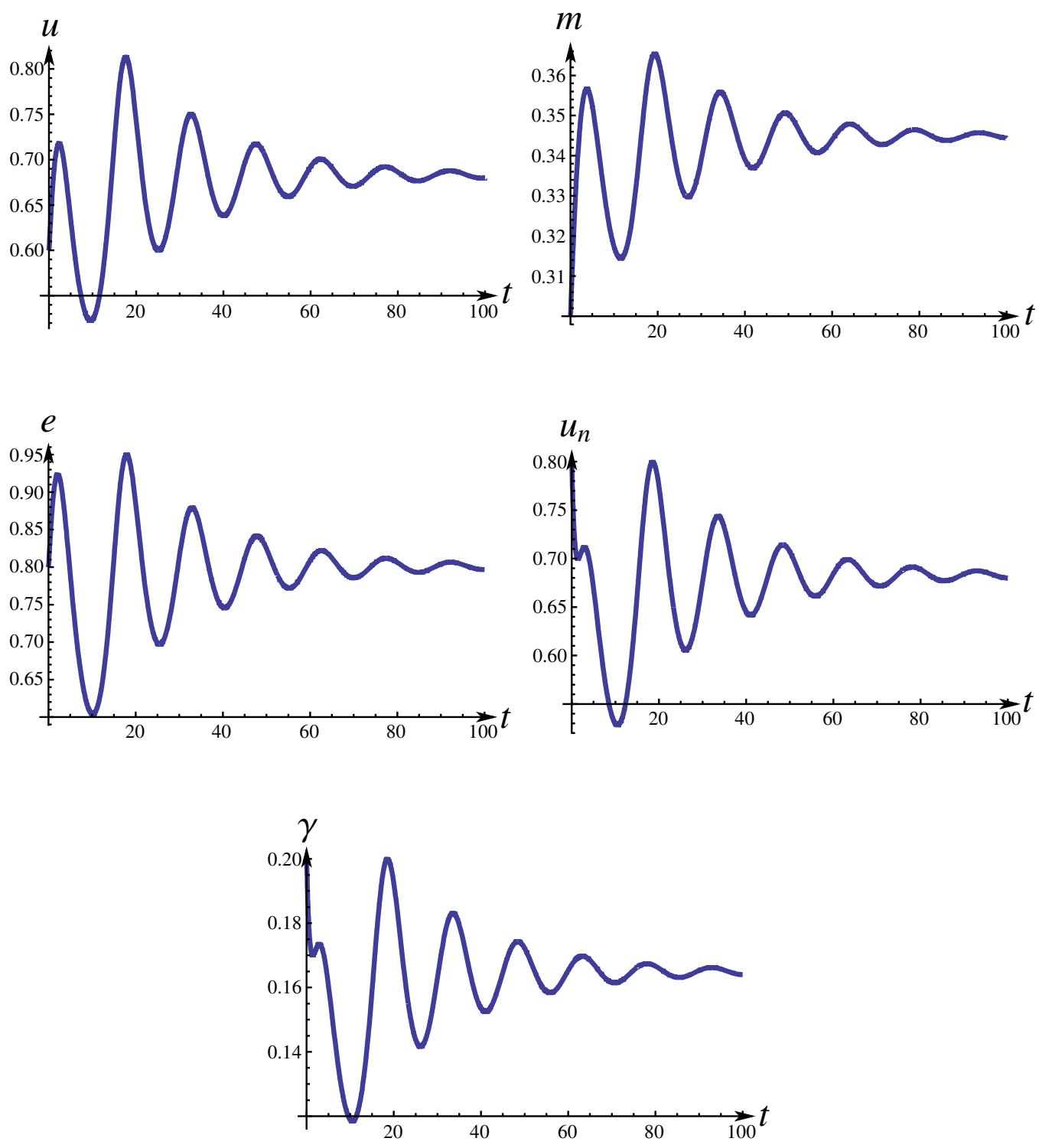

Figure 2: Cyclical fluctuations in the five-dimensional system 


\section{Appendix to "Conflict, Growth, Distribution, and Employment: A Long-Run Kaleckian Model”' (not for publication)}

\section{A-1: Derivation of the medium-run equilibrium profit share}

We derive the profit share in the medium-run equilibrium. The discriminant of equation (14) in the text is given by

$$
D=\Theta^{2}-4 s \varepsilon(A-n)=s^{2}\left(\gamma-\varepsilon u_{n}\right)^{2}+[s(A-n)-\varepsilon]^{2}+2 s\left(\gamma-\varepsilon u_{n}\right)[s(A-n)+\varepsilon]>0 .
$$

Hence, the quadratic equation (14) has the two real and distinct roots, $m_{1}$ and $m_{2}$. Let $m_{1}<m_{2}$. We have

$$
\begin{aligned}
& m_{1}+m_{2}=\frac{s\left(\gamma-\varepsilon u_{n}\right)+s(A-n)+\varepsilon}{s}>0, \\
& m_{1} m_{2}=\frac{(A-n) \varepsilon}{s}>0
\end{aligned}
$$

and thus, both $m_{1}$ and $m_{2}$ are positive. Rearranging the condition $s m-\varepsilon>0$, we obtain $m>\varepsilon / s$. Accordingly, $m$ has to satisfy this inequality. Let the left-hand side of equation (14) be $f(m)$. Because $f(\varepsilon / s)=-\left(\gamma-\varepsilon u_{n}\right)<0, m_{1}$ is smaller than $m=\varepsilon / s$, and $m_{2}$ is larger than $m=\varepsilon / s$. Therefore, $m_{2}$ is the medium-run equilibrium value of the profit share.

\section{A-2: Medium-run comparative statics analysis}

We conduct a comparative statics analysis in the medium-run equilibrium.

-A rise in $A$

The effect of a rise in $A$ on $m$ is as follows. Totally differentiating $\dot{m}=0$ and $\dot{e}=0$, we have

$$
\frac{d m}{d A}=\frac{1}{1-(\partial g / \partial m)}
$$

From the short-run analysis, we have $\partial g / \partial m<0$, and consequently, this derivative is positive. Therefore, a rise in $A$ increases the profit share.

The effect of a rise in $A$ on $e$ is as follows:

$$
\frac{d e}{d A}=\frac{\frac{\partial g / \partial m}{1-(\partial g / \partial m)}}{g_{a}^{\prime}(e)} .
$$

The denominator is positive and the numerator is negative, and consequently, this derivative is 
negative. Therefore, a rise in $A$ decreases the rate of employment.

The effect of a rise in $A$ on $u$ is opposite to the effect of a rise in $A$ on $m$ because $u$ is decreasing in $m$.

A rise in $s$

The effect of a rise in $s$ on $m$ is as follows:

$$
\frac{d m}{d s}=\frac{\partial g / \partial s}{1-(\partial g / \partial m)}
$$

The denominator is positive and the numerator is negative, and consequently, this derivative is negative. Therefore, a rise in $s$ decreases the profit share.

The effect of a rise in $s$ on $e$ is as follows:

$$
\frac{d e}{d s}=\frac{d m / d s}{g_{a}^{\prime}(e)} .
$$

The denominator is positive and the numerator is negative, and consequently, this derivative is negative. Therefore, a rise in $s$ decreases the rate of employment.

A rise in $n$

The effect of a rise in $n$ on $m$ is as follows:

$$
\frac{d m}{d n}=-\frac{1}{1-(\partial g / \partial m)} \text {. }
$$

The denominator is positive, and consequently, this derivative is negative. Therefore, a rise in $n$ decreases the profit share.

The effect of a rise in $n$ on $e$ is as follows:

$$
\frac{d e}{d n}=\frac{d m / d n}{g_{a}^{\prime}(e)} \text {. }
$$

The denominator is positive and the numerator is negative, and consequently, this derivative is negative. Therefore, a rise in $n$ decreases the rate of employment.

The effect of a rise in $n$ on $u$ is opposite to the effect of a rise in $n$ on $m$ because $u$ is decreasing in $m$.

\section{A-3: Endogenizing the target profit shares of workers and firms}

We explain how our results are modified if we endogenize the workers' target and the firms' target.

First, we assume that the target profit share of workers is a decreasing function of the 
growth rate of labor productivity.

$$
m_{w}=m_{w}\left(g_{a}\right), \quad m_{w}^{\prime}\left(g_{a}\right)<0 .
$$

Because $g_{a}=g_{a}(e)$ and $g_{a}^{\prime}(e)>0$, we can rewrite it as follows:

$$
m_{w}=m_{w}\left(g_{a}(e)\right)=m_{w}(e), \quad m_{w}^{\prime}(e)<0 .
$$

This equation relates the fruit of labor productivity gain to a wage claim.

Second, we assume that the target profit share of firms is an increasing function of the growth rate of labor productivity.

$$
m_{f}=m_{f}\left(g_{a}\right), \quad m_{f}^{\prime}\left(g_{a}\right)>0 .
$$

Because $g_{a}=g_{a}(e)$ and $g_{a}^{\prime}(e)>0$, we can rewrite it as follows:

$$
m_{f}=m_{f}\left(g_{a}(e)\right)=m_{f}(e), \quad m_{f}^{\prime}(e)>0 .
$$

This equation relates the fruit of labor productivity gain to a profit claim.

Using these equations, we obtain the following equation of motion for the profit share.

$$
\dot{m}=-(1-m)\left[m-\theta m_{f}(e)-(1-\theta) m_{w}(e)-g_{a}(e)\right],
$$

where the sing below the variables denotes the sign of the corresponding partial derivative of the variable. In this case, $J_{12}$ of the Jacobian matrix is modified as follows:

$$
J_{12}=\frac{\partial \dot{m}}{\partial e}=(1-m)\left[\theta m_{f}^{\prime}(e)+(1-\theta) m_{w}^{\prime}(e)+g_{a}^{\prime}(e)\right],
$$

which is evaluated at the medium-run equilibrium.

When we do not endogenize the targets, the first and second terms in the square bracket of the right-hand side will be zero, which yields $J_{12}>0$ with $g_{a}^{\prime}(e)>0$, as in the text.

When we endogenize the targets, the first term is positive and the second term is negative. If the negative effect of $m_{w}^{\prime}(e)<0$ is smaller than the other two positive effects, we have $J_{12}>0$, and accordingly, the foregoing analysis remains unchanged. If, on the other hand, the negative effect of $m_{w}^{\prime}(e)<0$ is larger than the other two positive effects, we have $J_{12}>0$, which is likely to yield $\operatorname{det} \mathbf{J}<0$. In this case, the medium-run equilibrium corresponds to the saddle point, and hence, it is unstable.

If the medium-run equilibrium is stable, that is, $J_{12}>0$ and hence $\operatorname{det} \mathbf{J}>0$, results of comparative statics analysis remain unchanged. For example, the effect of a rise in the bargaining power of firms leads to 


$$
\frac{d e}{d \theta}=\frac{m_{f}(e)-m_{w}(e)}{\frac{g_{a}^{\prime}(e)}{g^{\prime}(m)}-\left[\theta m_{f}^{\prime}(e)+(1-\theta) m_{w}^{\prime}(e)+g_{a}^{\prime}(e)\right]}=\frac{m_{f}(e)-m_{w}(e)}{\frac{g_{a}^{\prime}(e)}{g^{\prime}(m)}-\frac{J_{12}}{1-m}} .
$$

Here, we assume $m_{f}(e)>m_{w}(e)$ as in the text. Then, the numerator of the right-hand side is positive. In our model, the short-run equilibrium corresponds to wage-led growth, and hence, we have $g^{\prime}(m)<0$. From $g_{a}^{\prime}(e)>0$ and $J_{12}>0$, the denominator of the right-hand side is negative. It follows that we have $d e / d \theta<0$ : a rise in the bargaining power of firms lowers the equilibrium employment rate, which is the same as that in the text. Similar arguments hold for other derivatives.

\section{A-4: Derivation of equation (20)}

We derive equation (20) in the text. In the long-run equilibrium, $u^{* *}$ and $u_{n}$ are equalized, and accordingly, $u_{n}$ and $\gamma$ satisfy the following relation:

$$
\frac{\gamma-\varepsilon u_{n}}{\operatorname{sm}^{* *}\left(u_{n}, \gamma\right)-\varepsilon}=u_{n} \text {. }
$$

Because we know that the long-run equilibrium value of the profit share is given by $m^{* * *}=\gamma+A-n$, we can replace $m^{* *}$ with $m^{* * *}$ as follows:

$$
\frac{\gamma-\varepsilon u_{n}}{s(\gamma+A-n)-\varepsilon}=u_{n}
$$

The long-run equilibrium values of $u_{n}$ and $\gamma$ have to satisfy equation (3-2), which is a rewritten form of equation (20). Substituting equation (15) in the text into $m^{* *}$ of equation (3-1), we obtain

$$
\frac{\gamma-\varepsilon u_{n}}{\frac{\left[s\left(\gamma-\varepsilon u_{n}\right)+s(A-n)+\varepsilon\right]+\sqrt{\left[s\left(\gamma-\varepsilon u_{n}\right)+s(A-n)+\varepsilon\right]^{2}-4 s \varepsilon(A-n)}}{2}-\varepsilon}=u_{n} .
$$

Using numerical simulations, we confirm that equation (3-2) yields exactly the same results as equation (3-3). 
A-5: Convergence and path-dependency in the five-dimensional system with relatively small initial values (Case 1)

Time paths of the five variables in Case 1 of table 3 are as follows:
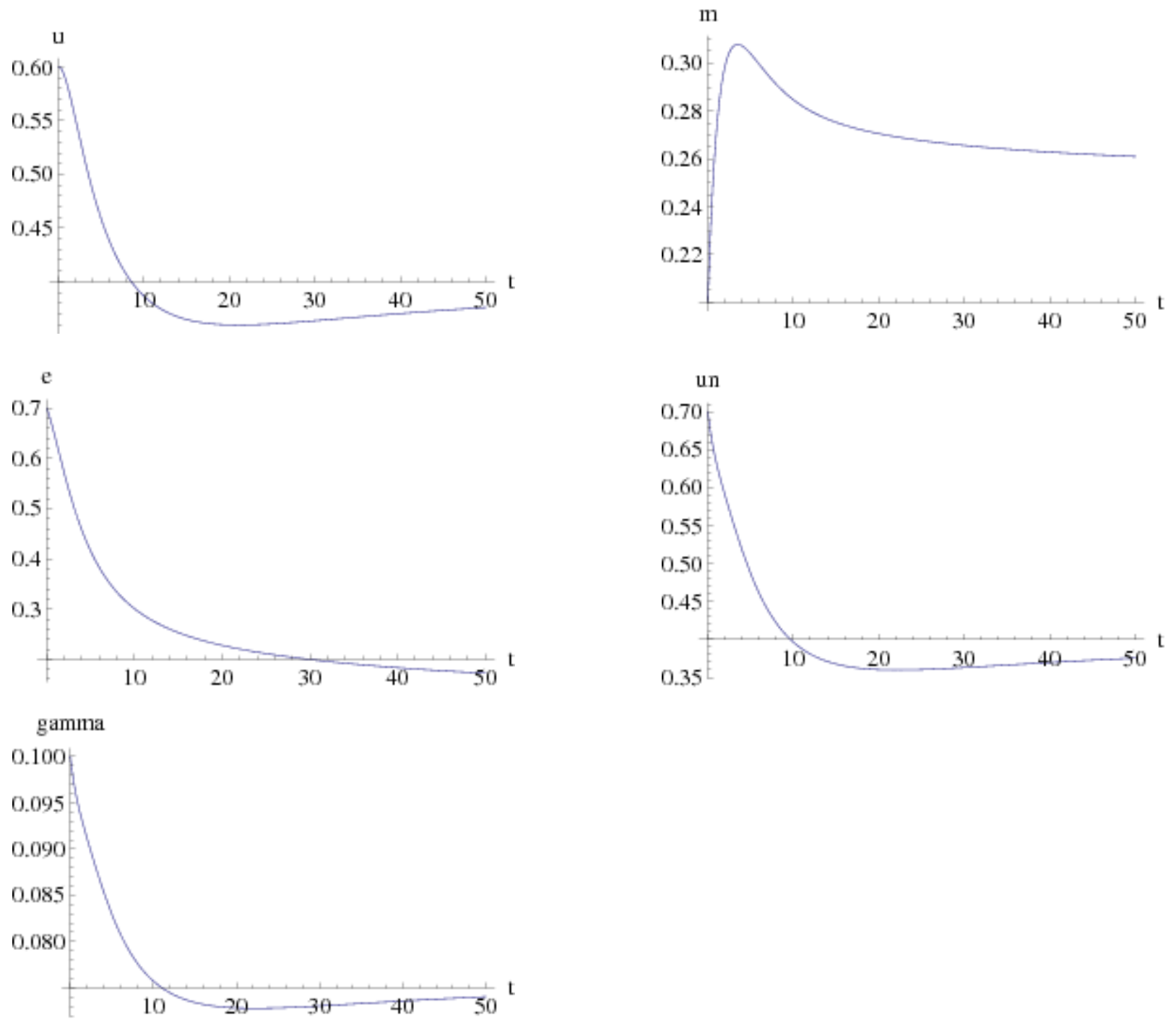
A-6: Convergence and path-dependency in the five-dimensional system with relatively large initial values (Case 2)

Time paths of the five variables in Case 2 of table 3 are as follows:
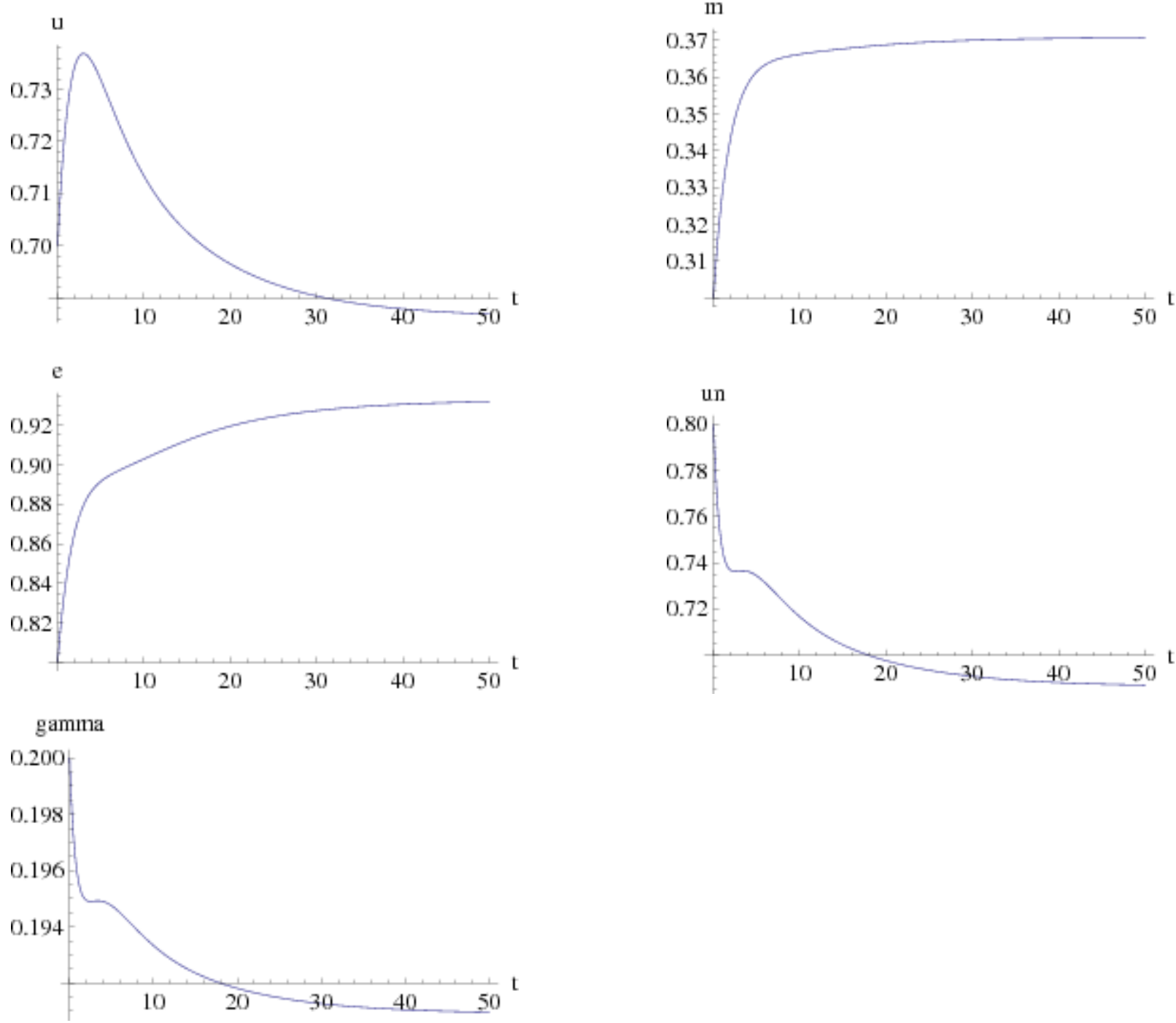